\title{
Alleviate Seed Ageing Effects in Silybum marianum by Application of Hormone Seed Priming
}

\author{
Seyed Ata SIADAT ${ }^{1 *}$, Seyed Amir MOOSAVI ${ }^{2}$, Mehran SHARAFIZADEH ${ }^{3}$ \\ ${ }^{1}$ Department of Agronomy and Plant Breeding, Dezful branch, Islamic Azad University, Iran; Seyedatasiadat@yahoo.com (*correspondingauthor) \\ ${ }^{2}$ Department of Agronomy, Faculty of Agriculture, Ramin Agriculture and Natural Resources Univeristy, Abwaz, Iran \\ ${ }^{3}$ Safiabad Agriculture Research Center, Dezful, Iran
}

\begin{abstract}
Most of the medicinal plants have seed dormancy or poor seed germination. This is due to their natural compounds or their morphological structure. Existence of such mechanisms makes the seeds able to endure harsh environments, stressful conditions or natural ageing. Different seed enhancement treatments were proposed in order to improve seed germination. In this study, it was examined Silybum marianum seed longevity ( 0,48 and 72 hour of ageing) and the response to seed priming when using different concentrations of cytokinin $(50,200,350$ and $500 \mathrm{ppm})$ and different duration of treatment $(8,12$ and 24 hour). Results revealed that ageing could be successfully alleviated using seed priming. Seed ageing significantly affected seed germination and vigour. Application of cytokinin considerably improved seed vigour in aged and non aged seeds. The most effective seed priming treatment was with 500 ppm cytokine, with the duration of 24 hours. In conclusion, it can be recommend the used of plant growth regulators like cytokine, as a good priming agent, to recover losses of seed quality and improve germination characteristics.
\end{abstract}

Keywords: cytokine, germination, longevity, seed dormancy, seed ageing

\section{Introduction}

Seed priming has been widely used by many researchers to improve germination parameters in different crops. Most of medical plants are known to have hard germinating seeds. In order to improve plant emergence and crop establishment it is critical for seedling for be as vigorous as possible and to achieve this goal, scientists try different seed enhancement techniques, like seed priming (Bewley, 1997). Seed priming is started by imbibition and ended before testa rupture. It means that in seed priming, seed imbibition is completed; lag phase of seed germination, which is the most important phase, is completed, but seeds are prohibited to enter into the next phase. During the second phase of seed germination most of metabolic pathways and recovering processes start, so that the embryo is assured that everything is prepared for successful germination (Varierl et al., 2010).

Silybum marianum is a medicinal plant which is under high consideration nowadays due to its medicinal properties and metabolites. The plant extract is widely used for treating liver disorders, protecting liver from hypotoxic drugs and chronic hepatitis. Flavonoids which are extracted from its seeds are high in antioxidants and could protect cell damage by oxidative stress.

Among all different environmental factors which affects seed vigour and viability, seed ageing is a common stress, especially in soil seed bank or seed storage rooms. Seed ageing decreases quality of seedling growth and germination traits of many plants (Eisvand, 2010). Different physiological processes are active during ageing. Changes in anti-oxidative activity, reverse mobilization and embryo weakening are severe effects of seed ageing. Application of accelerated ageing test was firstly used by Delouche and Baskin to measure seed vigor for different plant species. Lipid peroxidation may be the main key factor of decreasing seed quality by seed ageing. It is initiated with generation of free oxygen radicals (Gille and Joenje, 1991; Larson, 1997; McDonald, 1999). There are studies concluding that the application of plant growth regulators like $\mathrm{GA}_{3}$, ethephone, infused ether, kinetin could improve seed storage and germination characteristics like germination rate in different plant species.

In this study it was investigated Silybum marianum seed germination and seedling growth parameters. It was studied if hormone seed priming may recover side effects of seed ageing in regard to germination and growth parameters.

\section{Materials and Methods}

Mill thistle (Silybum marianum L.) seeds were obtained from Pakan Bazr Company, Isfahan, Iran. Three accelerated aging regimes were performed by placing the seeds in the incubator at a temperature of $40^{\circ} \mathrm{C}$ and relative humidity of 90 to $95 \%$ for 0,48 and 72 hours.

For each aging treatment, about $400 \mathrm{~g}$ of pure Milk thistle seeds were scattered within a vacuum container on wire screens; the container was filled with distilled water $(70 \%$ of the total container volume). The containers were placed in an incubator at the fixed temperature of $40^{\circ} \mathrm{C}$.

Seeds were subjected to phytohormone seed priming using 
different concentrations of cytokinin (50, 200,350 and 500 ppm); duration of seed priming was another experimental factor that was considered for 8,12 and 24 hours. The experiment was performed using a completely randomized design with four replications.

The standard germination test was performed by placing 25 seeds on top of two Whatman no. 1 filter papers in $10 \mathrm{~cm}$ petri dishes. All petri dishes were moistened with $10 \mathrm{ml}$ of distilled water and covered with plastic bags in order to reduce the water evaporation and then all petri dishes moved to germinator with 25 ${ }^{\circ} \mathrm{C}$ temperature, in dark condition.

Seeds were observed daily until day $7^{\text {th }}$; seeds were considered germinated when the radicle length reached $2 \mathrm{~mm}$.

Investigated parameters were the final germination percentage, mean daily germination (MDG) (Scott et al., 1984), coefficient of velocity of germination (CVG) (Maguire, 1962), root length, stem length and seedling vigour.

$$
\begin{aligned}
& \mathrm{MDG}=\frac{\text { Final germinationPercentage }}{\text { Days to reach the highest germination }} \\
& \mathrm{CVG}=\frac{G 1+G 2+G 3+\ldots+G n}{(1 * G 1)+(2 * G 2)+(3 * G 3)+\ldots(n * G n)}
\end{aligned}
$$

Where:

G1-GN: Numbers of germinated seed from the first day of experiment to the day $n$.

Statistical data analysis was performed by using Minitab, 16, MSTAT-C and Microsoft Excel 2010 software.

\section{Results}

The analyses of variance showed that there was a significant difference among the different ageing treatments and this was even more enhanced at higher duration of ageing. Using seed priming reduced some negative effects of ageing, compared to non-primed seeds.

\section{Germination percentage}

Analysis of variance showed that all main effects of different ageing treatments, cytokinin concentrations and duration of seed priming respectively, were significant. Among interaction effects, priming (cytokinin*time) was highly significant. Increased duration of the ageing treatment resulted in lower germination properties. Ageing condition negatively affected cell membrane,, therefore oxidative stress severely damaged the cells. Cytokinin promoted cell division and probably it activated some responsible recovery genes, and all eviate ageing side effects (Fig. 1). Increased ageing duration decreased the germination percentage (Table 1). Results showed that the most effective priming treatment for increasing germination percentage was 12 hour soaking seeds in $500 \mathrm{ppm}$ cytokinin, while the combination of $50 \mathrm{ppm}$ cytokinin with 8 hour of seed priming did not show significant difference (Table 2).

\section{Coefficient of velocity of germination ( $C V G$ )}

Analysis of variance showed that all priming treatments produced significant differences among all groups. Ageing severely decreased CVG, which indicated that germination rate was negatively affected. The longer the ageing period, the lower germination rate index CVG was (Fig. 2). Generally, lower durations of seed priming with cytokinin produced rapid germination and higher CVG value (Table 1). The

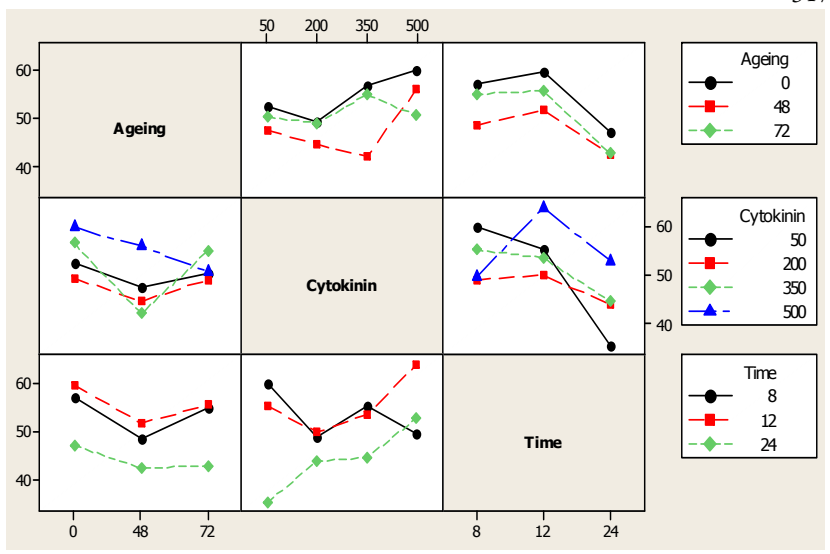

Fig. 1. Interaction plot of seed germination percentage under hormone seed priming and ageing treatments

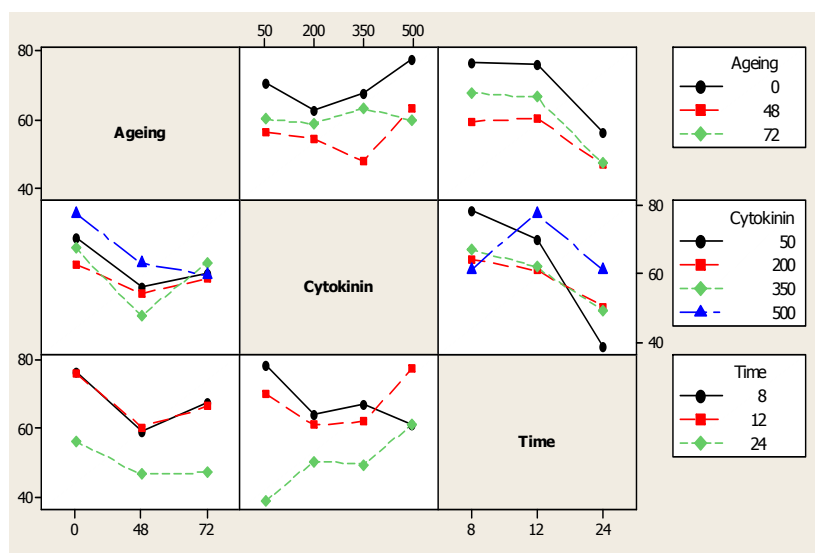

Fig. 2. Interaction plot of coefficient velocity of seed germination under hormone seed priming and ageing treatments

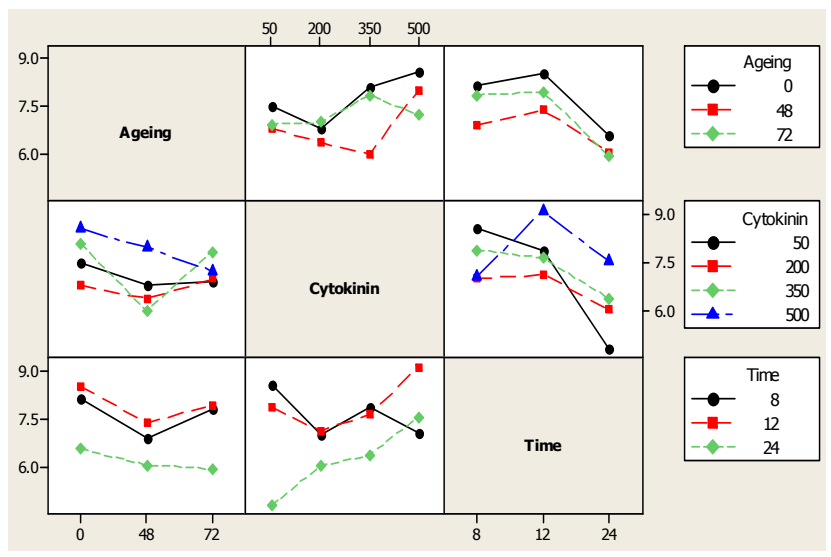

Fig. 3. Interaction plot of mean daily germination under hormone seed priming and ageing treatments

highest CVG value was observed in priming treatment with $50 \mathrm{ppm}$ cytokinin for 8 hours, which was slightly higher than that obtained for primed seeds with $500 \mathrm{ppm}$ cytokinin for 12 hour (Table 2).

Seed ageing decreased mean daily germination. Seed priming increased mean daily germination in aged and non-aged seeds (Fig. 3).

The highest MDG was observed in priming treatment of 12 h soaking in 500 ppm cytokinin, while the lowest priming 
318

Table 1. Mean comparison of ageing duration, priming duration and cytokinin concentrations on germination properties of Silybum marianum

\begin{tabular}{|c|c|c|c|c|c|c|c|c|c|c|c|c|}
\hline & \multicolumn{2}{|c|}{ Germination(\%) } & \multicolumn{2}{|c|}{ CVG } & \multicolumn{2}{|c|}{ MDG } & \multicolumn{2}{|c|}{ Rootlength $(\mathrm{cm})$} & \multicolumn{2}{|c|}{ Stem length $(\mathrm{cm})$} & \multicolumn{2}{|c|}{ Vigor } \\
\hline Ageing(hour) & Mean & Grouping & Mean & Grouping & Mean & Grouping & Mean & Grouping & Mean & Grouping & Mean & Grouping \\
\hline 0 & 54.646 & A & 69.821 & A & 7.75 & A & 7.646 & A & 9.273 & A & 933.56 & A \\
\hline 72 & 51.229 & $\mathrm{AB}$ & 55.518 & B & 6.798 & B & 7.871 & A & 9.344 & A & 825.85 & B \\
\hline 48 & 47.583 & $\mathrm{~B}$ & 60.526 & B & 7.257 & $\mathrm{AB}$ & 7.965 & A & 9.177 & A & 883.96 & $\mathrm{AB}$ \\
\hline \multicolumn{13}{|l|}{ Time (hour) } \\
\hline 8 & 53.5 & A & 67.996 & A & 7.643 & A & 7.165 & $\mathrm{C}$ & 9.802 & A & 911.73 & A \\
\hline 12 & 55.729 & A & 67.785 & A & 7.963 & A & 7.696 & B & 9.315 & B & 953.16 & A \\
\hline 24 & 44.229 & B & 50.083 & B & 6.198 & B & 8.621 & A & 8.677 & $\mathrm{C}$ & 778.49 & B \\
\hline \multicolumn{13}{|c|}{ Cytokinin (ppm) } \\
\hline 50 & 50.222 & $\mathrm{AB}$ & 62.551 & $\mathrm{AB}$ & 7.09 & B & 8.611 & A & 9.011 & $\mathrm{C}$ & 836.7 & B \\
\hline 200 & 47.667 & B & 58.698 & B & 6.728 & B & 7.419 & $\mathrm{BC}$ & 9.383 & $\mathrm{AB}$ & 828.03 & B \\
\hline 350 & 51.194 & $\mathrm{AB}$ & 59.646 & $\mathrm{AB}$ & 7.32 & $\mathrm{AB}$ & 7.958 & B & 9.058 & $\mathrm{BC}$ & 842.73 & B \\
\hline 500 & 55.528 & A & 66.926 & A & 7.935 & A & 7.319 & C & 9.606 & A & 1017.04 & A \\
\hline
\end{tabular}

Means that do not share the same letter are significantly different.

Table 2. Mean comparison of priming duration and cytokinin concentrations on germination properties of Silybum marianum

\begin{tabular}{|c|c|c|c|c|c|c|c|c|c|c|c|c|c|}
\hline & & \multicolumn{2}{|c|}{ Germination (\%) } & \multicolumn{2}{|c|}{ CVG } & \multicolumn{2}{|c|}{ MDG } & \multicolumn{2}{|c|}{ Rootlength $(\mathrm{cm})$} & \multicolumn{2}{|c|}{ Stem length $(\mathrm{cm})$} & \multicolumn{2}{|c|}{ Vigor } \\
\hline \multicolumn{2}{|c|}{ Cytokinin*Time } & Mean & Grouping & Mean & Grouping & Mean & Grouping & Mean & Grouping & Mean & Grouping & Mean & Grouping \\
\hline 50 & 8 & 60 & $\mathrm{AB}$ & 78.788 & A & 8.571 & $\mathrm{AB}$ & 7.667 & $\mathrm{C}$ & 9.792 & $\mathrm{AB}$ & 1049.03 & $\mathrm{AB}$ \\
\hline 50 & 12 & 55.333 & $\mathrm{ABC}$ & 70.038 & $\mathrm{ABC}$ & 7.905 & $\mathrm{ABC}$ & 7.417 & $\mathrm{C}$ & 9.142 & B & 917.47 & $\mathrm{ABC}$ \\
\hline 50 & 24 & 35.333 & $\mathrm{D}$ & 38.826 & E & 4.794 & $\mathrm{D}$ & 7.175 & $\mathrm{C}$ & 8.1 & C & 543.6 & $\mathrm{D}$ \\
\hline 200 & 8 & 49 & $\mathrm{BCD}$ & 64.371 & $A B C D$ & 7 & $\mathrm{BC}$ & 6.858 & C & 9.767 & $\mathrm{AB}$ & 816.77 & BCD \\
\hline 200 & 12 & 50 & $\mathrm{ABC}$ & 61.122 & $\mathrm{CD}$ & 7.143 & BC & 7.883 & BC & 9.25 & $\mathrm{AB}$ & 862.4 & $\mathrm{ABC}$ \\
\hline 200 & 24 & 44 & $C D$ & 50.6 & $\mathrm{DE}$ & 6.04 & $C D$ & 9.133 & B & 9.133 & B & 804.93 & $B C D$ \\
\hline 350 & 8 & 55.333 & $\mathrm{ABC}$ & 67.289 & $\mathrm{ABC}$ & 7.905 & $\mathrm{ABC}$ & 7.117 & C & 9.967 & A & 945.6 & $\mathrm{ABC}$ \\
\hline 350 & 12 & 53.583 & $\mathrm{ABC}$ & 62.109 & BCD & 7.663 & $\mathrm{ABC}$ & 7.583 & $\mathrm{C}$ & 9.125 & B & 898.13 & $\mathrm{ABC}$ \\
\hline 350 & 24 & 44.667 & $\mathrm{CD}$ & 49.54 & $\mathrm{DE}$ & 6.392 & $\mathrm{CD}$ & 7.258 & $\mathrm{C}$ & 8.083 & C & 684.47 & $\mathrm{CD}$ \\
\hline 500 & 8 & 49.667 & $\mathrm{ABCD}$ & 61.538 & $\mathrm{BCD}$ & 7.095 & BC & 7.017 & $\mathrm{C}$ & 9.683 & $\mathrm{AB}$ & 835.53 & BC \\
\hline 500 & 12 & 64 & A & 77.872 & $\mathrm{AB}$ & 9.143 & A & 7.9 & $\mathrm{BC}$ & 9.742 & $\mathrm{AB}$ & 1134.63 & A \\
\hline 500 & 24 & 52.917 & $\mathrm{ABC}$ & 61.367 & $C D$ & 7.568 & $\mathrm{ABC}$ & 10.917 & A & 9.392 & $\mathrm{AB}$ & 1080.95 & $\mathrm{AB}$ \\
\hline
\end{tabular}

Means that do not share the same letter are significantly different.

Table 3. Mean comparison of ageing duration and cytokinin concentrations on germination properties of Silybum marianum

\begin{tabular}{|c|c|c|c|c|c|c|c|c|c|c|c|c|c|}
\hline & & \multicolumn{2}{|c|}{ Germination (\%) } & \multicolumn{2}{|c|}{ CVG } & \multicolumn{2}{|c|}{ MDG } & \multicolumn{2}{|c|}{ Root length $(\mathrm{cm})$} & \multicolumn{2}{|c|}{ Stem length $(\mathrm{cm})$} & \multicolumn{2}{|c|}{ Vigor } \\
\hline & okinin & Mean & Grouping & Mean & Grouping & Mean & Grouping & Mean & Grouping & Mean & Grouping & Mean & Grouping \\
\hline 0 & 50 & 52.667 & $\mathrm{ABC}$ & 70.871 & $\mathrm{AB}$ & 7.524 & $\mathrm{ABC}$ & 7.675 & $\mathrm{ABCD}$ & 9.167 & $\mathrm{ABC}$ & 890.73 & $\mathrm{ABC}$ \\
\hline 0 & 200 & 49.333 & $\mathrm{ABC}$ & 62.932 & $\mathrm{ABC}$ & 6.802 & $\mathrm{ABC}$ & 7.358 & $\mathrm{CD}$ & 9.183 & $\mathrm{ABC}$ & 823.2 & $\mathrm{ABC}$ \\
\hline 0 & 350 & 56.667 & $\mathrm{AB}$ & 67.697 & $\mathrm{AB}$ & 8.106 & $\mathrm{AB}$ & 7.358 & CD & 9.133 & $\mathrm{ABC}$ & 942.9 & $\mathrm{ABC}$ \\
\hline 0 & 500 & 59.917 & A & 77.782 & A & 8.568 & A & 8.192 & $A B C D$ & 9.608 & $\mathrm{AB}$ & 1077.42 & A \\
\hline 48 & 50 & 47.667 & $\mathrm{ABC}$ & 56.539 & $\mathrm{BC}$ & 6.81 & $\mathrm{ABC}$ & 7.058 & D & 9.1 & $\mathrm{BC}$ & 776.67 & $\mathrm{C}$ \\
\hline 48 & 200 & 44.667 & $\mathrm{BC}$ & 54.455 & $\mathrm{BC}$ & 6.381 & $\mathrm{BC}$ & 8.408 & $\mathrm{ABC}$ & 9.475 & $\mathrm{ABC}$ & 794.3 & $\mathrm{BC}$ \\
\hline 48 & 350 & 42 & C & 47.873 & $\mathrm{C}$ & 6 & $\mathrm{C}$ & 7.075 & D & 8.875 & $\mathrm{BC}$ & 671.6 & $\mathrm{C}$ \\
\hline 48 & 500 & 56 & $\mathrm{ABC}$ & 63.206 & $\mathrm{ABC}$ & 8 & $A B$ & 8.942 & A & 9.925 & A & 1060.83 & $\mathrm{AB}$ \\
\hline 72 & 50 & 50.333 & $\mathrm{ABC}$ & 60.242 & $\mathrm{BC}$ & 6.937 & $\mathrm{ABC}$ & 7.525 & $\mathrm{BCD}$ & 8.767 & C & 842.7 & $\mathrm{ABC}$ \\
\hline 72 & 200 & 49 & $\mathrm{ABC}$ & 58.705 & $\mathrm{BC}$ & 7 & $\mathrm{ABC}$ & 8.108 & $A B C D$ & 9.492 & $\mathrm{ABC}$ & 866.6 & $\mathrm{ABC}$ \\
\hline 72 & 350 & 54.917 & $\mathrm{ABC}$ & 63.367 & $\mathrm{ABC}$ & 7.854 & $\mathrm{ABC}$ & 7.525 & $\mathrm{BCD}$ & 9.167 & $\mathrm{ABC}$ & 913.69 & $\mathrm{ABC}$ \\
\hline 72 & 500 & 50.667 & $\mathrm{ABC}$ & 59.789 & $\mathrm{BC}$ & 7.238 & $\mathrm{ABC}$ & 8.7 & $\mathrm{AB}$ & 9.283 & $\mathrm{ABC}$ & 912.87 & $\mathrm{ABC}$ \\
\hline
\end{tabular}

Means that do not share the same letter are significantly different.

treatment was $24 \mathrm{~h}$ soaking seeds in $50 \mathrm{ppm}$ cytokinin (Table 2).

Seed priming significantly affected root elongation in Silybum marianum. Results revealed that longer roots were produced after seed priming treatment of soaking seeds with 500 ppm cytokinin for 24 hour (Table 2). Cytokinin promoted root elongation and positively alleviated ageing side effects on root growth (Fig. 4).

Threeway interaction was significant for stem growth of Silybum marianum. Performance of lower concentration of cytokinin combined with lower priming durations was better, while higher cytokinin concentrations did not show the same trend (Fig. 5). The increase in ageing duration resulted in a reduction of the stem growth, thus to alleviation this side effect of ageing, more cytokinin concentration should be applied to

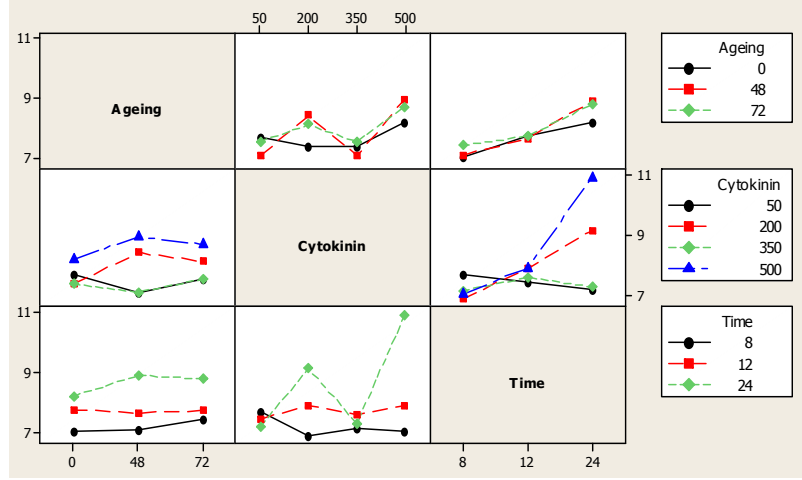

Fig. 4. Interaction plot of root length under hormone seed priming and ageing treatments 
Table 4. Mean comparison of ageing and priming durations on germination properties of Silybum marianum

\begin{tabular}{|c|c|c|c|c|c|c|c|c|c|c|c|c|c|}
\hline \multirow[b]{2}{*}{ Ageing } & \multirow[b]{2}{*}{ Time } & \multicolumn{2}{|c|}{ Germination (\%) } & \multicolumn{2}{|c|}{ CVG } & \multicolumn{2}{|c|}{ MDG } & \multicolumn{2}{|c|}{ Root length $(\mathrm{cm})$} & \multicolumn{2}{|c|}{ Stem length $(\mathrm{cm})$} & \multicolumn{2}{|c|}{ Vigor } \\
\hline & & Mean & Grouping & Mean & Grouping & Mean & Grouping & Mean & Grouping & Mean & Grouping & Mean & Grouping \\
\hline 0 & 8 & 57 & $\mathrm{AB}$ & 76.724 & A & 8.143 & $\mathrm{AB}$ & 7.012 & $\mathrm{D}$ & 9.806 & A & 960.5 & $\mathrm{AB}$ \\
\hline 0 & 12 & 59.75 & A & 76.344 & A & 8.536 & A & 7.737 & $\mathrm{BCD}$ & 9.331 & $A B$ & 1029.28 & A \\
\hline 0 & 24 & 47.187 & $\mathrm{BC}$ & 56.393 & BCD & 6.571 & $C D$ & 8.188 & $\mathrm{ABC}$ & 8.681 & $\mathrm{BC}$ & 810.91 & $\mathrm{AB}$ \\
\hline 48 & 8 & 48.5 & $\mathrm{ABC}$ & 59.413 & $B C D$ & 6.929 & $B C D$ & 7.081 & $\mathrm{D}$ & 9.844 & A & 821.15 & $A B$ \\
\hline 48 & 12 & 51.75 & $\mathrm{ABC}$ & 60.477 & $\mathrm{BC}$ & 7.393 & $A B C D$ & 7.638 & $C D$ & 9.313 & $A B$ & 886.02 & $A B$ \\
\hline 48 & 24 & 42.5 & $\mathrm{C}$ & 46.665 & $\mathrm{D}$ & 6.071 & D & 8.894 & A & 8.875 & $\mathrm{BC}$ & 770.37 & $\mathrm{~B}$ \\
\hline 72 & 8 & 55 & $A B$ & 67.851 & $A B$ & 7.857 & $\mathrm{ABC}$ & 7.4 & $C D$ & 9.756 & A & 953.55 & $\mathrm{AB}$ \\
\hline 72 & 12 & 55.688 & $A B$ & 66.535 & $\mathrm{AB}$ & 7.962 & $\mathrm{ABC}$ & 7.713 & $C D$ & 9.3 & $A B$ & 944.17 & $A B$ \\
\hline 72 & 24 & 43 & $\mathrm{C}$ & 47.192 & $C D$ & 5.953 & $\mathrm{D}$ & 8.781 & $A B$ & 8.475 & $\mathrm{C}$ & 754.17 & B \\
\hline
\end{tabular}

Means that do not share the same letter are significantly different.

Table 5. Mean comparison of interaction of ageing, priming duration and cytokinin concentrations on germination percentage, CVG and MDG of Silybum marianum

\begin{tabular}{|c|c|c|c|c|c|c|c|c|}
\hline \multirow[b]{2}{*}{ Ageing } & \multirow[b]{2}{*}{ Cytokinin } & \multirow[b]{2}{*}{ Time } & \multicolumn{2}{|c|}{ Germination (\%) } & \multicolumn{2}{|c|}{ CVG } & \multicolumn{2}{|c|}{ MDG } \\
\hline & & & Mean & Grouping & Mean & Grouping & Mean & Grouping \\
\hline 0 & 50 & 8 & 61 & $\mathrm{ABCD}$ & 86.536 & $\mathrm{AB}$ & 8.714 & $\mathrm{ABCD}$ \\
\hline 0 & 50 & 12 & 61 & A B C D & 81.038 & A B C & 8.714 & A B C D \\
\hline 0 & 50 & 24 & 36 & C D & 45.038 & DEF & 5.143 & DE \\
\hline 0 & 200 & 8 & 49 & A B C D & 68.036 & A B C D E & 7 & A B C DE \\
\hline 0 & 200 & 12 & 53 & A B C D & 69.037 & A B C D E & 7.571 & A B C D E \\
\hline 0 & 200 & 24 & 46 & A B C D & 51.723 & CDEF & 5.834 & BCDE \\
\hline 0 & 350 & 8 & 65 & A B C & 82.538 & A B C & 9.286 & A B C \\
\hline 0 & 350 & 12 & 56 & A B C D & 65.015 & A B C D E & 8 & A B C D \\
\hline 0 & 350 & 24 & 49 & A B C D & 55.539 & B C D E F & 7.034 & A B C D E \\
\hline 0 & 500 & 8 & 53 & A B C D & 69.787 & $\mathrm{ABCDE}$ & 7.571 & A B C D E \\
\hline 0 & 500 & 12 & 69 & A & 90.288 & A & 9.857 & A \\
\hline 0 & 500 & 24 & 57.75 & A B C D & 73.272 & A B C D E & 8.275 & A B C D \\
\hline 48 & 50 & 8 & 57 & A B C D & 70.789 & A B C D E & 8.143 & A B C D \\
\hline 48 & 50 & 12 & 49 & A B C D & 58.539 & ABCDEF & 7 & A B C DE \\
\hline 48 & 50 & 24 & 37 & B C D & 40.29 & EF & 5.286 & DE \\
\hline 48 & 200 & 8 & 45 & $\mathrm{ABCD}$ & 56.288 & BCDEF & 6.429 & A B CDE \\
\hline 48 & 200 & 12 & 52 & $\mathrm{ABCD}$ & 62.539 & A B C DEF & 7.429 & $\mathrm{ABCDE}$ \\
\hline 48 & 200 & 24 & 37 & B C D & 44.539 & DEF & 5.286 & DE \\
\hline 48 & 350 & 8 & 47 & A B C D & 58.538 & A B CDEF & 6.714 & $\mathrm{ABCDE}$ \\
\hline 48 & 350 & 12 & 40 & $A B C D$ & 44.54 & DEF & 5.714 & B C D E \\
\hline 48 & 350 & 24 & 39 & B C D & 40.54 & EF & 5.571 & CDE \\
\hline 48 & 500 & 8 & 45 & $A B C D$ & 52.039 & CDEF & 6.429 & A B C D E \\
\hline 48 & 500 & 12 & 66 & A B & 76.289 & A B C D & 9.429 & A B \\
\hline 48 & 500 & 24 & 57 & A B C D & 61.29 & A B CDEF & 8.143 & A B C D \\
\hline 72 & 50 & 8 & 62 & A B C D & 79.038 & A B C & 8.857 & A B C D \\
\hline 72 & 50 & 12 & 56 & $A B C D$ & 70.538 & A B C D E & 8 & A B C D \\
\hline 72 & 50 & 24 & 33 & D & 31.149 & F & 3.953 & E \\
\hline 72 & 200 & 8 & 53 & A B C D & 68.787 & A B C DE & 7.571 & A B C DE \\
\hline 72 & 200 & 12 & 45 & $A B C D$ & 51.789 & CDEF & 6.429 & A B C DE \\
\hline 72 & 200 & 24 & 49 & A B C D & 55.539 & BCDEF & 7 & $\mathrm{ABCDE}$ \\
\hline 72 & 350 & 8 & 54 & A B C D & 60.79 & A B CDEF & 7.714 & A B C D E \\
\hline 72 & 350 & 12 & 64.75 & A B C & 76.773 & A B C D & 9.275 & A B C \\
\hline 72 & 350 & 24 & 46 & A B C D & 52.539 & C D E F & 6.571 & A B C D E \\
\hline 72 & 500 & 8 & 51 & $A B C D$ & 62.788 & A B CDEF & 7.286 & A B C DE \\
\hline 72 & 500 & 12 & 57 & A B C D & 67.039 & A B C D E & 8.143 & A B C D \\
\hline 72 & 500 & 24 & 44 & A B C D & 49.54 & CDEF & 6.286 & A B C D E \\
\hline
\end{tabular}

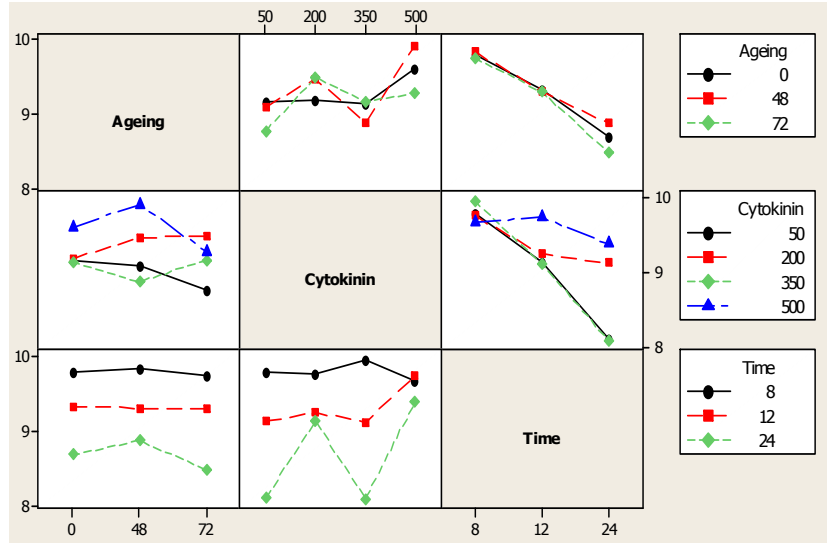

Fig. 5. Interaction plot of stem length under hormone seed priming and ageing treatments

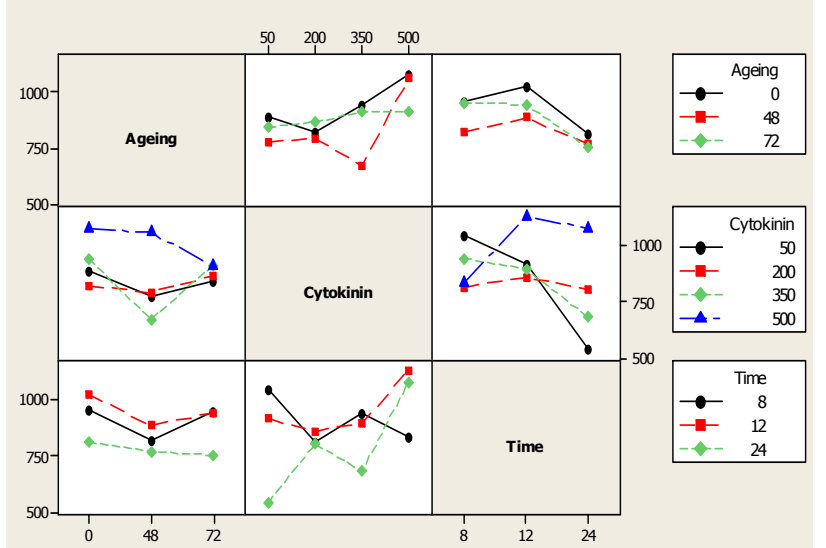

Fig. 6. Interaction plot of vigor index under hormone seed priming and ageing treatments 
320

Table 6. Mean comparison of interaction of ageing, priming duration and cytokinin concentrations on root length $(\mathrm{cm})$, stem length $(\mathrm{cm})$ and seed vigor of Silybum marianum

\begin{tabular}{|c|c|c|c|c|c|c|c|c|}
\hline \multirow[b]{2}{*}{ Ageing } & \multirow[b]{2}{*}{ Cytokinin } & \multirow[b]{2}{*}{ Time } & \multicolumn{2}{|c|}{ Root length $(\mathrm{cm})$} & \multicolumn{2}{|c|}{ Stem length $(\mathrm{cm})$} & \multicolumn{2}{|c|}{ Vigor } \\
\hline & & & Mean & Grouping & Mean & Grouping & Mean & Grouping \\
\hline 0 & 50 & 8 & 7.575 & CDE & 9.8 & $\mathrm{~A}$ & 1060.3 & $\mathrm{AB}$ \\
\hline 0 & 50 & 12 & 7.65 & C DE & 9.075 & A B C & 1019.3 & A B C \\
\hline 0 & 50 & 24 & 7.8 & C D E & 8.625 & A B C D & 592.6 & B C \\
\hline 0 & 200 & 8 & 6.7 & E & 9.575 & A B & 794.5 & A B C \\
\hline 0 & 200 & 12 & 7.125 & E & 9.025 & $\mathrm{ABC}$ & 872.4 & A B C \\
\hline 0 & 200 & 24 & 8.25 & CDE & 8.95 & $\mathrm{ABC}$ & 802.7 & $\mathrm{ABC}$ \\
\hline 0 & 350 & 8 & 7 & E & 9.875 & A & 1094.5 & A B \\
\hline 0 & 350 & 12 & 8.6 & B C D E & 9.475 & A B & 1020.4 & A B C \\
\hline 0 & 350 & 24 & 6.475 & E & 8.05 & B C D & 713.8 & A B C \\
\hline 0 & 500 & 8 & 6.775 & E & 9.975 & A & 892.7 & A B C \\
\hline 0 & 500 & 12 & 7.575 & CDE & 9.75 & A & 1205 & A \\
\hline 0 & 500 & 24 & 10.225 & $\mathrm{ABC}$ & 9.1 & A B C & 1134.55 & A B \\
\hline 48 & 50 & 8 & 7.25 & E & 9.725 & A & 967 & A B C \\
\hline 48 & 50 & 12 & 6.925 & $\mathrm{E}$ & 9.025 & A B C & 784.7 & A B C \\
\hline 48 & 50 & 24 & 7 & E & 8.55 & A B C D & 578.3 & B C \\
\hline 48 & 200 & 8 & 6.8 & E & 9.8 & A & 750.1 & $\mathrm{ABC}$ \\
\hline 48 & 200 & 12 & 8.3 & C DE & 9.225 & $\mathrm{AB}$ & 912.6 & A B C \\
\hline 48 & 200 & 24 & 10.125 & $\mathrm{ABCD}$ & 9.4 & A B & 720.2 & $\mathrm{ABC}$ \\
\hline 48 & 350 & 8 & 6.775 & E & 10.175 & A & 796.9 & A B C \\
\hline 48 & 350 & 12 & 7.25 & E & 8.975 & A B C & 647 & A B C \\
\hline 48 & 350 & 24 & 7.2 & E & 7.475 & C D & 570.9 & B C \\
\hline 48 & 500 & 8 & 7.5 & $\mathrm{DE}$ & 9.675 & $\mathrm{AB}$ & 770.6 & $\mathrm{ABC}$ \\
\hline 48 & 500 & 12 & 8.075 & C DE & 10.025 & A & 1199.8 & A \\
\hline 48 & 500 & 24 & 11.25 & A B & 10.075 & A & 1212.1 & A \\
\hline 72 & 50 & 8 & 8.175 & C DE & 9.85 & $\mathrm{~A}$ & 1119.8 & $\mathrm{AB}$ \\
\hline 72 & 50 & 12 & 7.675 & $\mathrm{CDE}$ & 9.325 & A B & 948.4 & $\mathrm{ABC}$ \\
\hline 72 & 50 & 24 & 6.725 & E & 7.125 & $\mathrm{D}$ & 459.9 & C \\
\hline 72 & 200 & 8 & 7.075 & E & 9.925 & $\mathrm{~A}$ & 905.7 & $\mathrm{ABC}$ \\
\hline 72 & 200 & 12 & 8.225 & C DE & 9.5 & A B & 802.2 & A B C \\
\hline 72 & 200 & 24 & 9.025 & A B C DE & 9.05 & $\mathrm{ABC}$ & 891.9 & A B C \\
\hline 72 & 350 & 8 & 7.575 & CDE & 9.85 & A & 945.4 & A B C \\
\hline 72 & 350 & 12 & 6.9 & E & 8.925 & A B C & 1026.98 & A B C \\
\hline 72 & 350 & 24 & 8.1 & CDE & 8.725 & A B C D & 768.7 & A B C \\
\hline 72 & 500 & 8 & 6.775 & E & 9.4 & A B & 843.3 & A B C \\
\hline 72 & 500 & 12 & 8.05 & C DE & 9.45 & A B & 999.1 & A B C \\
\hline 72 & 500 & 24 & 11.275 & A & 9 & $\mathrm{ABC}$ & 896.2 & A B C \\
\hline
\end{tabular}

seeds (Table 6). The results are in agreement with other data, as seed priming significantly improved final germination percentage (GP), mean germination time (MGT), mean daily germination (MDG), daily germination speed (DGS) and germination rate index (GI) of Canola under water stress (Behrouzyar and Yarnia, 2014).

Seed vigour decreased for aged seeds, but seed priming positively improved seed vigour. The higher the cytokinin concentration, the more seed vigour was achieved (Fig. 6). The highest seed vigour was achieved for priming treatment of $500 \mathrm{ppm}$ cytokinin with the duration of 48 hours. Results showed that, not only seed priming alleviated ageing side effects, but also it increased seed vigour compared to nonaged seeds (Table 6). Seed priming enhanced germination percentage (GP), germination index (GI) and mean germination rate (MGR) in aged tomato seeds. Seedling characteristics such as radicle and shoot length were increased compared with unprimed aged tomato seeds (Zhang et al., 2014).

\section{Discussion}

Seed longevity is the potential of seed survival in the surrounding environment and it will initiate just by the time that seeds have passed to their maturity level. Seed longevity allows plant population to endure long after the disappearance of the parent plants, so it directly influences genetic disturbance and diversity. Seed longevity also is important in designing long-term weed management programs, as well as good knowledge of plant population dynamics (Long et al., 2014). Agreement of the ecophysiological mechanisms of seed longevity is critical for researchers to anticipate how long seeds can survive in the soil seed bank under different climatic conditions. Seed ageing lead to vigour and viability losses and perhaps this is due to losses in cell membrane integrity, because of lipid peroxidation by free radicals, lack of ATP and reduction in protein synthesis (Gidrol et al., 1988; Gille and Joenje, 1991; Larson, 1997).

Seed treatment could help to recover ageing effects on seeds and seed priming is an effective seed treatment to overcome ageing. As it has been cleared in many crops, during imbibition repair process of seeds are initiated and thus seed priming improves germination characteristics (McDonald, 1999; Imran et al., 2013; Prom-u-thai et al., 2012; Yadav et al., 2011).

Upon placing dry seeds in water, the uptake of water will be initiated in three stages (Bewley, 1997). At the first stage, namely imbibition, water uptake is initiated rapidly due to lower water potential of the seeds. During the second stage, germination is started by activation of different physiological activities including protein synthesis, translation of new mRNAs etc. The third has a rapid water trend, when germination will be completed. Therefore, successful seed priming is highly dependent on first and second stages of seed 
germination, when seeds are starting to absorb water from the surrounding environment (Varierl et al., 2010).

Seed priming activates or synthesis many of enzymes that are involved in remobilization of seed reservoirs like storage proteins. HSP proteins are also synthesized during seed priming. These proteins are stress related proteins, as they could protect seed proteins from natural ageing (Kester et al., 1997; Varierl et al., 2010).

Plant growth regulators have been widely used as priming agent on different plant seeds in order to alleviate the negative effects of stress conditions (Afzal et al., 2005; Egamberdieva, 2009; Eisvand et al., 2010; Gadallah, 1999). Salicylic acid, giberlline, auxin and cytokinin are widely used in seed enhancement experiments. Cytokine is one of the important plant hormones that is involved in controlling cell division, photomorphogenesis, chloroplast development and root growth (Brault and Maldiney, 1999; Davies, 1995). Optimal concentration of cytokinin exhibited favourable effects on germination and seedling growth (Gadallah, 1999; Iqbal and Ashraf, 2006). It has been reported that seed priming with 10 ppm kinetin increased final germination percentage, germination index, shoot length and seedling fresh weight of two tomato cultivars as compared to control (Nawaz et al., 2013).

\section{Conclusions}

The current experiment suggested that cytokine plays critical roles in plant responses to ageing and it can be concluded that hormone priming with $500 \mathrm{ppm}$ cytokinin significantly increased seed vigour in both aged and non aged seeds. Seed vigour of primed seeds was not significantly different from non aged seeds. Therefore, hormonal priming with cytokine is recommended as a safe technique to relieve the negative effects of Silybum marianum seed deterioration.

\section{References}

Afzal I, Basra SMA, Iqbal A (2005). The effects of seed soaking with plant growth regulators on seedling vigor of wheat under salinity stress. Journal of Stress Physiology \& Biochemistry 1(1):6-14.

Behrouzyar EK, Yarnia M (2014). Effect of ethanol, methanol, zinc, manganese and boron seed priming on ageing, seed germination and physiological characteristics in canola under water deficit stress. Research on Crops 15(1):116-121.

Bewley JD (1997). Seed germination and dormancy. The Plant Cell 9(7):1055.

Brault M, Maldiney R (1999). Mechanisms of cytokinin action. Plant Physiology and Biochemistry 37(5):403-412.

Davies PJ (1995). The plant hormones: their nature, occurrence, and functions. In: Plant hormones. Springer pp 1-12.

Egamberdieva D (2009). Alleviation of salt stress by plant growth regulators and IAA producing bacteria in wheat. Acta Physiologiae Plantarum 31(4):861-864.

Eisvand HR, Alizadeh MA, Fekri A (2010). How hormonal priming of aged and nonaged seeds of bromegrass affects seedling physiological characters. Journal of New Seeds 11(1):52-64.
Gadallah MAA (1999). Effects of proline and glycinebetaine on Vicia faba responses to salt stress. Biologia Plantarum 42(2):249-257.

Gidrol X, Noubhani A, Mocquot B, Fournier A, Pradet A (1988). Effect of accelerated aging on protein synthesis in two legume seeds. Plant Physiology and Biochemistry 26(3):281-288.

Gille JJP, Joenje H (1991). Biological significance of oxygen toxicity: An introduction. Membrane Lipid Oxidation 3:1-32.

Imran M, Mahmood A, Römheld V, Neumann G (2013). Nutrient seed priming improves seedling development of maize exposed to low root zone temperatures during early growth. European Journal of Agronomy 49:141-148.

Iqbal M, Ashraf M (2006). Wheat seed priming in relation to salt tolerance: growth, yield and levels of free salicylic acid and polyamines. In: Annales Botanici Fennici Vol 43. Helsinki: Societas Biologica Fennica Vanamo pp 250-259.

Kester ST, Geneve RL, Houtz RL (1997). Priming and accelerated ageing affect L-isoaspartyl methyltransferase activity in tomato (Lycopersicon esculentum Mill.) seed. Journal of Experimental Botany 48(4):943-949.

Larson RA (1997). Naturally occurring antioxidants. CRC Press.

Long RL, Gorecki MJ, Renton M, Scott JK, Colville L, Goggin DE, Commander LE, Westcott DA et al. (2014). The ecophysiology of seed persistence: a mechanistic view of the journey to germination or demise. Biological Reviews 90(1):31-59.

Maguire JD (1962). Seed of germination-aid in selection and evaluation for seedling emergence and vigor. Crop Science 2:176-177.

McDonald MB (1999). Seed deterioration: physiology, repair and assessment. Seed Science and Technology 27(1):177-237.

Nawaz A, Amjad M, Khan SM, Afzal I, Ahmed T, Iqbal Q, Iqbal J (2013). Tomato seed invigoration with cytokinins. Journal of Animal and Plant Sciences 23(1):121-128.

Prom-u-thai C, Rerkasem B, Yazici A, Cakmak I (2012). Journal of Plant Nutrition and Soil Science 175(3):482-488.

Scott SJ, Jones RA, Willams WA (1984). Review of data analysis methods for seed germination. Crop Science 24:1192-1199.

Varierl A, Vari AK, Dadlani M (2010). The subcellular basis of seed priming. Current Science 99(4).

Yadav PV, Kumari M, Ahmed Z (2011). Seed priming mediated germination improvement and tolerance to subsequent exposure to cold and salt stress in capsicum. Res J Seed Sci 4:125-136.

Zhang M, Wang Z, Yuan L, Yin C, Cheng J, Wang L, Huang J, Zhang $\mathrm{H}$ (2014). Osmopriming improves tomato seed vigor under aging and salinity stress. African Journal of Biotechnology 11(23):63056311. 